diem, together with douches, every day, twice a day. Gymnastics and avoidance of all intellectual work or excitement. If the disease resist this treatment, he employs the oxide of zinc, associated with the bromide of potash. The oxide is given in doses of 2 decigrams per diem, in pills, as a maximal dose; but then the bromide should not be administered beyond 4 grams daily. Each pill of the oxide should contain I cgm., and one may begin by administering two pills twice a day, increasing by I centigram per diem until one reaches $20 \mathrm{cgms}$, the maximal dose; in young girls he adds extract of valerian to the zinc preparation. He claims that it is rare that chorea can resist this treatment methodically carried out. Simon employs rest in bed, revulsion to the upper portion of the spine, by means of mustard poultices, hot frictions and dry cups, hot-air baths, given in bed, every two days. Internally, he administers the tincture of aconite and cisuta, ro to 5 drops in twenty-four hours; if fever be present, the sulphate of quinine. Antipyrin he puts great confidence in. Methodic gymnastics are of great service as after-treatment (La Semaine médicale, No. 13,1892 ).

The eclectics claim results from actea racemosa-black colzosh (Translator).

F. H. P.

\title{
NOTE RELATIVE TO THE BUFFALO LITHIA WATER.
}

There is a point in relation to the therapeutical efficacy of the Buffalo Lithia Water which has not as yet, I think, received sufficient attention. It is well known that many cases of diseases of the nervous system are complicated with lithæmia, and unless this condition is removed a cure is very often retarded and not frequently entirely prevented. It is quite commonly the case that in cerebral congestion producing insomnia, nervous prostration resulting from over-mental work or much emotional disturbance, and in epilepsy (to say nothing of many cases of insanity) an excess of uric acid in the blood is often observed. This state appears to be altogether independent of the character of the food; for no matter how careful the physician may be in regard to the diet of his patient the lithrmic condition continues. I have tried to overcome this persistence by the use of phosphate of ammonia and other so-called solvents for uric acid, but without notable effect. 
Several years ago, however, I began to treat such cases with Buffalo Lithia Water, with a result that was astonishing to me as it was beneficial to the patient, so that now in all cases of nervous diseases under my charge in which there is an excess of uric acid in the blood, I use the Buffalo Lithia Water in large quantities. By this I mean that I do not have the patient drink merely a tumbler or two in the course of the day, but that I flood him, so to speak, with the water, making him drink a gallon or even more, in the twenty-four hours. By this course the urine after a few days ceases to deposit uric acid crystals on standing, the morbid irritability of the patient disappears, the tongue becomes clean, the wandering pains in the head are abolished, and the system is rendered much more amenable to the special treatment which may be necessary for the cure of the disease from which the patient suffers.

I have tried carbonate of lithia, dissolved in water, in various proportions; but it certainly does not, in cases to which I refer, have the same effect as Buffalo Lithia Water.-William A. HammoND, M.D.

Washington, D.C., Jan. 25, 1892.

\section{Soxtety aperoxts.}

NEW YORK NEUROLOGICAL SOCIETY.

Meeting of March 1,189 .

The President, Dr. L. C. Grar, in the chair.

\section{HYSTERIA IN A CHILD.}

Dr. W. M. LESZYNSKY presented a patient, a young girl, whom he said he had brought to demonstrate that we did have in this country cases of hysteria in children. She was, he said, only thirteen years of age. Two and a half years ago, after fright from a dream, the hysterical symptoms had manifested themselves in paroxysms of laughing and crying. In I89I she had commenced to menstruate and had then begun to have convulsive seizures, which were usually worse at the menstrual 